\title{
Role of Operon $a^{a} o_{\text {so }}$-mutT in Antioxidant Defense in Streptococcus oligofermentans
}

\author{
Peng Zhou, Lei Liu, Huichun Tong*, Xiuzhu Dong* \\ State Key Laboratory of Microbial Resources, Institute of Microbiology, Chinese Academy of Sciences, Beijing, China
}

\begin{abstract}
Previously, we have found that an insertional inactivation of $a a^{\circ o}$, a gene encoding L-amino acid oxidase (LAAO), causes marked repression of the growth of Streptococcus oligofermentans. Here, we found that $a_{a o_{s o}}$ and mutT, a homolog of pyrophosphohydrolase gene of Escherichia coli, constituted an operon. Deletion of either gene did not impair the growth of S. oligofermentans, but double deletion of both $a a o_{s o}$ and mutT was lethal. Quantitative PCR showed that the transcript abundance of mutT was reduced for 13 -fold in the $a a_{s o}$ insertional mutant, indicating that gene polarity derived from the inactivation of $a_{a O_{S o}}$ attenuated the expression of mutT. Enzymatic assays were conducted to determine the biochemical functions of LAAO and MutT of S. oligofermentans. The results indicated that LAAO functioned as an aminoacetone oxidase [47.75 nmol $\mathrm{H}_{2} \mathrm{O}_{2}$ (min.mg protein) ${ }^{-1}$ ]; and MutT showed the pyrophosphohydrolase activity, which removed mutagens such as 8-oxo-dGTP. Like paraquat, $a a_{\text {so }}$ mutations increased the expression of SOD, and addition of aminoacetone (final concentration, $5 \mathrm{mM}$ ) decreased the mutant's growth by $11 \%$, indicating that the $a 0^{\circ o}$ mutants are under ROS stress. HPLC did reveal elevated levels of cytoplasmic aminoacetone in both the deletion and insertional gene mutants of $a a_{S o}$. Electron spin resonance spectroscopy showed increased hydroxyl radicals in both types of $a a o_{s o}$ mutant. This demonstrated that inactivation of $a a_{S o}$ caused the elevation of the prooxidant aminoacetone, resulting the cellular ROS stress. Our study indicates that the presence of both LAAO and MutT can prevent endogenous metabolites-generated ROS and mutagens. In this way, we were able to determine the role of the $a a_{S_{s o}}-$ mutT operon in antioxidant defense in S. oligofermentans.
\end{abstract}

Citation: Zhou P, Liu L, Tong H, Dong X (2012) Role of Operon $a a_{s_{0}}-$ mutT in Antioxidant Defense in Streptococcus oligofermentans. PLoS ONE 7(5): e38133. doi:10.1371/journal.pone.0038133

Editor: Paul Sumby, The Methodist Hospital Research Institute, United States of America

Received February 19, 2012; Accepted May 4, 2012; Published May 30, 2012

Copyright: (C) 2012 Zhou et al. This is an open-access article distributed under the terms of the Creative Commons Attribution License, which permits unrestricted use, distribution, and reproduction in any medium, provided the original author and source are credited.

Funding: This study was supported by the China National Science Foundation under grant 30870042 . The funders had no role in study design, data collection and analysis, decision to publish, or preparation of the manuscript.

Competing Interests: The authors have declared that no competing interests exist.

*E-mail: dongxz@im.ac.cn (XD); Tonghuichun@im.ac.cn (HT)

\section{Introduction}

Oxidative stress is encountered universally by all organisms living in an oxygen environment. Oxygen is a relatively inert molecule in nature. However, when it is channeled to electrontransfer reactions, especially inside the cells that experience autooxidation of reduced enzyme cofactors, oxygen is converted into deleterious reactive oxygen species (ROS). Due to the higher energy that results from the presence of unpaired valence-shell electrons, ROS can cause severe damage to various biological macromolecules, especially DNA. This leads to mutagenesis, tumorigenesis, and aging [1,2].

Organisms have developed various means of resisting oxidative stress. Aerobes generally employ superoxide dismutase (SOD) and catalase cascades to eliminate cellular ROS. SOD dismutes superoxide anions as $\mathrm{H}_{2} \mathrm{O}_{2}$, which is then converted to $\mathrm{H}_{2} \mathrm{O}$ and $\mathrm{O}_{2}$ by catalase [3,4]. Anaerobes use the superoxide reductase (SOR)-dependent oxide decomposition pathway [5]. SOR reduces superoxide to $\mathrm{H}_{2} \mathrm{O}_{2}$ using reduced rubredoxin $\left(\mathrm{Rd}_{\mathrm{red}}\right)$ as the electron donor. Then, in concert with the non-heme peroxidase rubrerythrin, SOR reduces $\mathrm{H}_{2} \mathrm{O}_{2}$ to water. Intact $\mathrm{H}_{2} \mathrm{O}_{2}$ is not particularly deleterious to cells, but $\mathrm{H}_{2} \mathrm{O}_{2}$-derived hydroxyl radicals are indiscriminately damaging to the biological macromolecules. The conversion of $\mathrm{H}_{2} \mathrm{O}_{2}$ into radicals takes place through Fenton chemistry, which is activated by ferrous or copper ions [6-8]. Streptococci, which are facultative anaerobes, undergo fermentative metabolism to produce energy reserves. Streptococci do not possess catalase; instead, they have SOD and other oxidases. The formation of $\mathrm{H}_{2} \mathrm{O}_{2}$ is their main means of attenuating ROS damage [4,6]. Streptococcus oligofermentans is isolated from non-caries dental plaques in humans. It uses multiple pathways for the generation of $\mathrm{H}_{2} \mathrm{O}_{2}$ from oxygen [9]. These pathways include lactate oxidase (Lox) in oxidation of lactate to produce $\mathrm{H}_{2} \mathrm{O}_{2}$ [10]; L-amino acid oxidase (LAAO) to oxidize amino acids to $\mathrm{H}_{2} \mathrm{O}_{2}$ [11]; and a recently identified pyruvate oxidase (Pox) [12]. Lox and Pox contribute the majority of the $\mathrm{H}_{2} \mathrm{O}_{2}$ derived from glycolysis products [12], and LAAO generates lower levels of $\mathrm{H}_{2} \mathrm{O}_{2}$ when proteinaceous substances are present. Among streptococci, $S$. oligofermentans is one of those with the highest levels of tolerance to $\mathrm{H}_{2} \mathrm{O}_{2}$ [13]. This makes it a suitable model organism for the study of the mechanisms by which bacteria protect themselves against oxidative stress.

In a previous study on LAAO, we found that insertional inactivation of $a a o_{S o}$ caused severe reductions in the growth of $S$. oligofermentans [11]. To gain insight into the growth retardation, we performed a microarray assay and observed the differential expression of some genes in $a a_{0_{S o}}$ mutants, including the genes encoding manganese-dependent SOD ( $\operatorname{sod} A)$, DNA-binding ferritin-like protein, DNA repair enzyme, and NADH oxidase II (nox). Elevated $\mathrm{H}_{2} \mathrm{O}_{2}$ yield was detected in glucose-containing cultures of $a a_{S o}$ mutants. These results imply that inactivation of $a a o_{S o}$ causes an elevation of cellular ROS levels in S. oligofermentans. 
In the present study, we used genetic and biochemical analyses to find that LAAO is an authentic aminoacetone oxidase. It reduces the aminoacetone-derived ROS in vivo. Furthermore, $a a_{S o}$, by constituting an operon with $m u t T$, encoding a nucleotideoxide scavenging protein, develops a dual guard by reducing the production of cellular ROS and clearing the nucleotide oxides generated by ROS in streptococci. Thus, a novel antioxidant defense gene operon has been demonstrated.

\section{Results}

\section{Identification of the $a a o_{s o}-m u t T$ Operon in S. oligofermentans}

Previous studies have found that an insertional mutation of $a a 0_{S o}$ decreased the growth of $S$. oligofermentans to two thirds that of the wild-type strain [11]. This suggests that other inhibitory mechanisms might be present in addition to the $\mathrm{H}_{2} \mathrm{O}_{2}$ generated by LAAO. To evaluate possible inhibitive causes, we examined the genes that flanked $a a_{S o}$ in the genome of $S$. oligofermentans. As shown in Fig. 1A, a putative gene encoding MutT (Acc No. JQ083601), a homolog of pyrophosphohydrolase (8-oxo-dGTPase) in Escherichia coli [14], was located $19 \mathrm{bp}$ downstream of $a a 0_{S o}$. The two appear to constitute an operon. Then, using a reverse transcription-PCR assay, we determined that $a a_{S o}$ and $m u t T$ were cotranscribed (Fig. 1B). This confirmed the presence of the operon and coexpression of the two genes in S. oligofermentans.

\section{Role of Coexistence of $a a_{S o}$ and mutT in S.} oligofermentans

To determine the relative effects of $a a o_{S o}$ and $m u t T$ on the growth of $S$. oligofermentans, we constructed deletion mutants for each gene. As shown in Fig. S1, the deletion mutant $\triangle a a o_{S o}$ exhibited slightly slower growth than wild-type strain, and? $\Delta m u t T$ did not show any detectable decrease in growth. A complementary strain of the insertional mutant of $a a_{S_{o}}$ resumed growth (Fig. 2). However, we never obtained a double gene mutant, here denoted $\triangle a a o_{S o}-\Delta m u t T$, even under obligate anaerobic conditions by manipulating inside an anaerobic chamber. This implies that double mutation of the two genes may be lethal. To confirm this, we first cloned the $a a_{S_{o}}-m u t T$ operon into the $E$. coli-streptococcus shuttle-expression vector pDL278 and then introduced it into the wild-type strain of $S$. oligofermentans. Under this background, we readily deleted $a a_{S o}-m u t T$ from the chromosome. This mutant also carried a plasmid containing the operon, which showed growth patterns similar to the wild-type strain. To clarify the effect of an insertional mutation of $a a_{S_{o}}$ on the downstream mutT, we determined the expression of mut $T$ by quantitative PCR and found a 13-fold reduction in transcript abundance relative to that of the wild-type strain, but in the $\Delta a a_{S_{S o}}$ strain, the deletion mutant, mut $T$ expression was similar to the wild-type (Table 1). This indicates that $a a 0_{S o}$ inactivation caused a gene-polarity effect on $m u t T$. This insertional mutant was designated $\Delta a a_{S_{S o}}-m u t T$, and the suppression of growth was predicted to be related to the loss of MutT activity. Taken together, the coexistence of $a a o_{s o}$ and $m u t T$

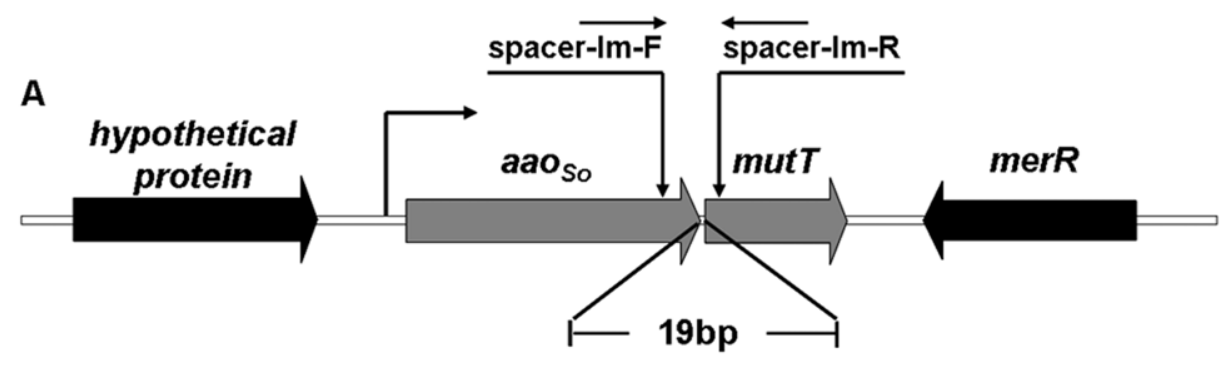

B

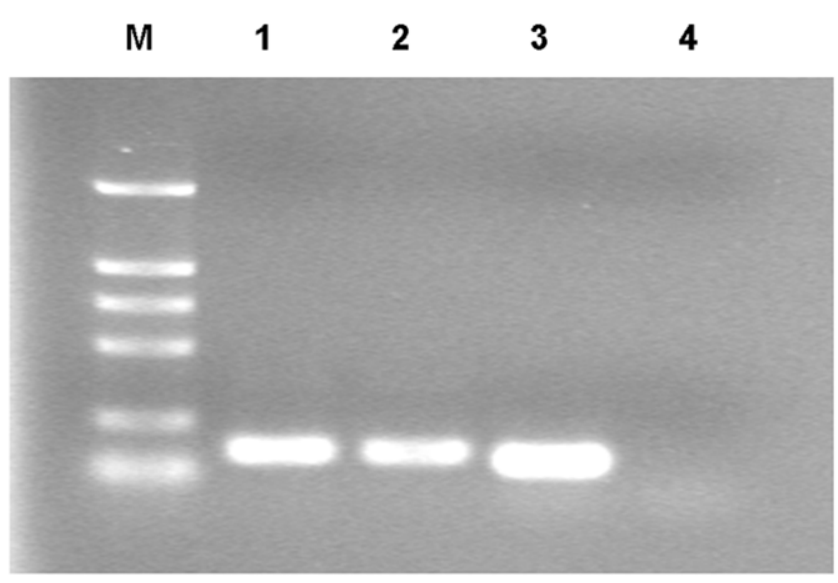

Figure 1. Cotranscription of $a a_{S o}$ and mutT in S. oligofermentans. (A) Schematic arrangement of $a a_{S o}$ and mutT in the genome of $S$. oligofermentans. (B) Agarose gel electrophoresis of the RT-PCR products for the intergenic spacer between $a_{a o_{S o}}$ and mutT. M, DL2000 marker; 1, PCR products amplified with primer spacer-Im-F and spacer-Im-R with gDNA as template; 2, PCR products amplified with primer spacer-Im- $\mathrm{F}$ and spacerIm-R using CDNA as template; 3, PCR products amplified with primer spacer-neg- $\mathrm{F}$ and spacer-neg-R with gDNA as template; 4, PCR products amplified with primer spacer-neg- $\mathrm{F}$ and spacer-neg-R with CDNA as template.

doi:10.1371/journal.pone.0038133.g001 


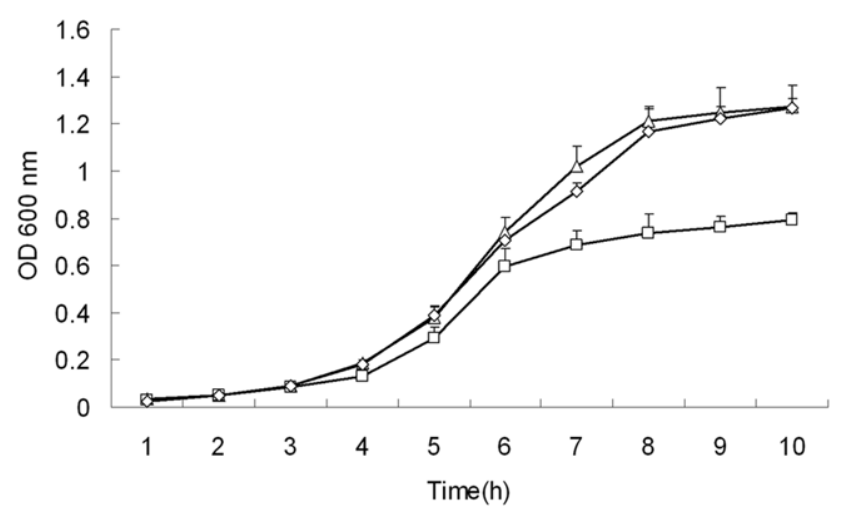

Figure 2. Growth of the wild-type, $\Delta a a o_{s o}-m u t T$ mutant of $S$. oligofermentans and its complementary strain in glucose. Strains were pre-cultured overnight in TYG medium, and 1:100 diluted with fresh TYG medium with the same $\mathrm{OD}_{600}$. Samples were taken every $1 \mathrm{~h}$ to detect $\mathrm{OD}_{600}$. Symbols: $\triangle$, wild-type strain; $\square, \Delta a a o_{S o}-$ mutT mutant; $\diamond, \Delta a a o_{S o}-m u t T$ complementary strain. The results are shown as the means $\pm S D$ of three independent experiments.

doi:10.1371/journal.pone.0038133.g002

seems to be essential for S. oligofermentans to carry out its normal physiological functions and even survive.

\section{LAAO Authentically Acts as an Aminoacetone Oxidase}

Enzymatic activity was assayed to clarify the function of LAAO. Our previous study showed that LAAO, an L-amino acid oxidase, mainly oxidizes seven kinds of amino acids to generate $\mathrm{H}_{2} \mathrm{O}_{2}$ [11]. Aminoacetone belongs to the amine family, and is determined as an electron donor in reactions involving active transition metals. So that it generates superoxide anions (ROS) from oxygen [15]. In human cells, semicarbazide-sensitive amine oxidase (SSAO) has been shown to remove aminoacetone by converting it to methylglyoxal (MG) and $\mathrm{H}_{2} \mathrm{O}_{2}[15,16]$. Given that aminoacetone is an analog of amino acids, we tested the enzymatic activity of the overexpressed LAAO on aminoacetone. By using $25 \mathrm{mM}$ of aminoacetone as substrate and air as an oxygen supply, we detected the production of $47.75 \mathrm{nmol} \mathrm{H}_{2} \mathrm{O}_{2}\left(\min \cdot \mathrm{mg}\right.$ protein) ${ }^{-1}$. In parallel, the activity of LAAO on lysine was only $2.22 \mathrm{nmol}$ $\mathrm{H}_{2} \mathrm{O}_{2}$ (min.mg protein) ${ }^{-1}$. Even when lysine concentration was increased to $100 \mathrm{mM}$, the activity was only $12.29 \mathrm{nmol}_{2} \mathrm{O}_{2}$ $(\min \cdot \mathrm{mg} \text { protein })^{-1}$. Similar low activities were detected for aspartic acid and glutamine (Fig. S2). This indicates that aminoacetone, not amino acids, is the preferred substrate of LAAO. However, unlike the described amine oxidases [17], LAAO did not catalyze benzylamine or methylamine conversion (data not shown).

Table 1. Transcript levels of mutT and mutX in the wild-type, $\Delta a a_{S o}$ and $\Delta a a o_{S_{o}}-$ mutT mutants of S. oligofermentans.

\begin{tabular}{|c|c|c|}
\hline \multirow[b]{2}{*}{ Strains } & \multicolumn{2}{|c|}{ The number of gene transcript* } \\
\hline & mutt & mutX \\
\hline wild-type & $0.66 \pm 0.15$ & $0.03 \pm 0.01$ \\
\hline$\Delta a a o_{S o}-m u t T$ & $0.05 \pm 0.01$ & ND \\
\hline$\Delta a a o_{S o}$ & $0.76 \pm 0.17$ & ND \\
\hline
\end{tabular}

Role of MutT in Elimination of Nucleotide Oxides in $S$. oligofermentans

Previous research has shown that dGTP can be readily oxidized to 8-oxo-dGTP by hydroxyl radicals, owing to its hypersensitivity to oxidation $[18,19]$. MutT of E. coli and its human homolog hMTH-1 protein hydrolyze 8-oxo-dGTP to pyrophosphate (PPi) and the harmless monophosphate 8-oxo-dGMP through a specific pyrophosphohydrolase (8-oxo-dGTPase) activity. Two mut T homologs, mut $T$ and $m u t X$, were found in the genome of $S$. oligofermentans. By using quantitative PCR, we determined that the transcript abundance of mut $T$ was about 20-fold higher than that of $m u t X$ (Table 1), similar to the results of our previous microarray assays (unpublished data).

Next, we determined the pyrophosphohydrolase activity of the purified MutT protein of $S$. oligofermentans in vitro. According to the activity of MutT of E. coli, synthetic 8-oxo-dGTP was used as the substrate in the enzymatic assay. Upon addition of the purified recombinant MutT protein from $S$. oligofermentans, reduction of 8oxo-dGTP levels was detected in the reaction mixture by HPLC (Fig. S3). This confirmed the pyrophosphohydrolase activity of $S$. oligofermentans MutT.

Effects of Inactivation of $a a_{S o}$ on the Regulation of sodA

Our previous microarray data identified increased mRNA from sodA, the only SOD-encoding gene in the S. oligofermentans genome, by the $a a_{S_{0}}$ mutant relative to the wild-type strain (unpublished data). As superoxide anion is the well-defined substrate of SOD, a link between LAAO protein and the cellular ROS level may exist. To test this hypothesis, quantitative PCR was used to assay the expression of sodA in the $a a 0_{S o}$ mutants of $S$. oligofermentans. As expected, higher transcript abundance of $\operatorname{sod} A$ was detected in $\triangle a a 0_{S o}-m u t T$ mutant $\left(76.63 \pm 13.62\right.$ copies) and $\Delta a a 0_{S o}$ mutant $(47.94 \pm 8.83$ copies $)$, relative to the wild-type strain $(13.11 \pm 3.02$ copies $)$. In addition, dramatic increases in Mn-SOD activity were observed in the early and middle log phase cultures of both mutants (Table 2). As sodA mRNA levels increase 3-fold in wild-type S. oligofermentans treated with the redox-cycling drug paraquat, which induces production of cellular endogenous superoxides (data not shown), we propose that the accumulation of superoxide anion increases the expression of $\mathrm{Mn}-\mathrm{SOD}$ in $\Delta a a o_{S o}-m u t T$ and $\Delta a a o_{S o}$ mutants.

We then constructed $\Delta s o d A$ and $\Delta a a o_{S o}-m u t T-\Delta s o d A$ mutants to confirm the superoxide-induced impairment of growth. As expected, no obvious decrease in growth was observed for $\Delta$ sodA mutant. However, a reduction in growth of nearly $70 \%$ was detected for the double mutant, $\Delta a a o_{S_{0}}-m u t T-\Delta$ sodA (Fig. S4). Whereas when grew under strictly anaerobic conditions, $\Delta a a 0_{S o}-m u t T$ mutant grew to a final $\mathrm{OD}_{600}$ of 1.2, similar to wild-type; and the growth of the mutant $\Delta a a 0_{S o}-m u t T-\Delta s o d A$ was restored to a level similar to that of the insertional mutant of $a a_{S o}$. This implies that superoxide anions may be the key to impairing the growth of $\Delta a a_{S o}-m u t T$ mutants.

Table 2. Mn-SOD activities of deletion and insertion mutants of S. oligofermentans $\mathrm{aao}_{\mathrm{So}}$.

\begin{tabular}{|c|c|c|c|}
\hline \multirow[b]{2}{*}{ Growth time } & \multicolumn{3}{|c|}{ Specific activity (U $\mathrm{mg}^{-1}$ protein)* } \\
\hline & wild-type & $\Delta a a o_{s o}$ & $\operatorname{daao}_{\text {so }}-\mathrm{mutT}$ \\
\hline $4 \mathrm{~h}$ & $2.55 \pm 0.68$ & $29.38 \pm 9.49$ & $34.89 \pm 4.92$ \\
\hline $6 \mathrm{~h}$ & $17.88 \pm 5.33$ & $43.43 \pm 10.54$ & $45.62 \pm 8.68$ \\
\hline
\end{tabular}

*Activity was detected using the method of pyrogallol auto-oxidation [40]. Data were obtained as means \pm SD from three independent experiments.

doi:10.1371/journal.pone.0038133.t002 

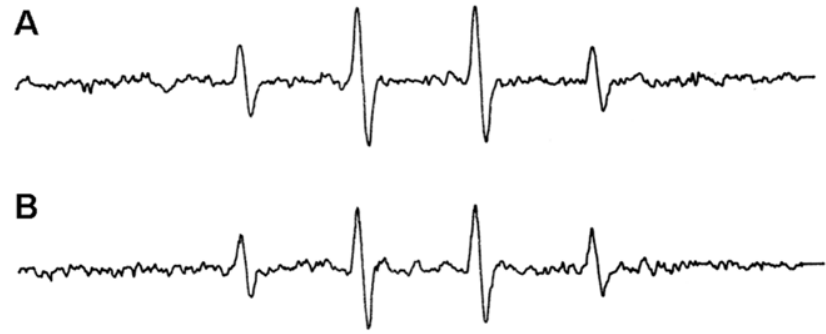

C

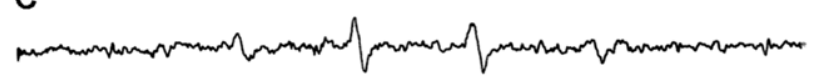

$2 \mathrm{mT}$

Figure 3. ESR spectra of the DMPO treated cells of $S$. oligofermentans. The characteristic 1:2:2:1 quartet of hydroxyl radical adduct of DMPO signals were shown in the DMPO-contained cell suspensions of (A) $\Delta a a o_{s o}-m u t T$ mutant, (B) $\Delta a a o_{s o}$ mutant, and (C) wild-type strain.

doi:10.1371/journal.pone.0038133.g003

\section{Effects of Mutation of $a^{a O_{s o}}$ on Cellular Levels of ROS}

To further evaluate the link between intracellular levels of ROS to LAAO, we measured the ROS levels in the $\Delta a a o_{S o}-m u t T, \Delta a a o_{S o}$ mutants and the wild-type strain. Considering that the accumulation of superoxide in cells is concomitant with abundant $\mathrm{H}_{2} \mathrm{O}_{2}$ generated by lactate oxidase and pyruvate oxidase, and either superoxide anion or $\mathrm{H}_{2} \mathrm{O}_{2}$ can cause the release of iron from proteins containing $[4 \mathrm{Fe}-4 \mathrm{~S}]^{2+}$ clusters, the Fenton reaction can cause an elevation in cellular hydroxyl radicals [20]. Therefore, hydroxyl radicals were assessed in S. oligofermentans strains using electron spin resonance (ESR) with 5,5-dimethyl-1-pyrroline- $\mathcal{N}$ oxide (DMPO) spin trap [21,22]. ESR spectroscopy is the most efficient and non-destructive technique for detecting chemical species that have unpaired electrons, such as the superoxide anion radical and hydroxyl radical. As a spin trap, DMPO has been frequently used to detect oxygen-centered radicals [22]. As shown in Fig. 3, intensive and characteristic 1:2:2:1 quartet of DMPO$\mathrm{OH}^{-}$adduct signal was obtained both in the $\Delta a a 0_{S o}-m u t T$ and $\Delta a a 0_{S o}$ mutants, but only a very weak signal was detected in the wild-type strain. This demonstrates that the $a a o_{S o}$ mutants were under a relatively high ROS stress.

\section{Effects of Mutation of $a a_{\text {so }}$ on Levels of Cytoplasmic Aminoacetone}

To evaluate the link between intracellular ROS and the inactivation of LAAO, based on the aminoacetone oxidase activity of LAAO, we assessed the intracellular accumulation of aminoacetone for the wild-type strain, $\Delta a a o_{S o}-m u t T$, and $\Delta a a o_{S o}$ mutants.
However, cytoplasmic aminoacetone was not detected in any strain, probably due to the instability of this compound. Using a method described by Kazachkov and $\mathrm{Yu}$, we generated a stable derivative of aminoacetone by addition of 9-fluorenylmethyl chloroformate-Cl (FMOC-Cl) to the cellular lysate [23]. As shown in Fig. S5, levels of aminoacetone as much as double that observed in wild-type $\left(76.8 \pm 12.5 \mathrm{pmol} \mathrm{mg}^{-1}\right.$ cell $)$ were determined in $\Delta a a_{S_{0}}-$ mut T mutant $\left(159.8 \pm 9.2 \mathrm{pmol} \mathrm{mg}^{-1}\right.$ cell $)$ and $\Delta a a_{0_{S_{o}}}$ strain (147.2 $\pm 21.4 \mathrm{pmol} \mathrm{mg}^{-1}$ cell). Similarly elevated levels were observed in semicarbazide-sensitive amine oxidase (SSAO)repressed human tissues [23]. This determines that LAAO has a function similar to that of amine oxidase in removing the endogenous aminoacetone.

Next, we added $5 \mathrm{mmol}$ aminoacetone to cultures of wild-type, $\Delta a a 0_{S o}-m u t T$ mutant, and $\Delta a a 0_{S o}$ mutant. It was found that aminoacetone reduced the growth of $\Delta a a_{0} S_{S o}-m u t T$ mutant by $11 \%$, but it increased the growth of the wild-type and $\Delta a a o_{S o}$ mutant by $10 \%$ (Table 3). Cellular aminoacetone can be derived from threonine or glycine [24], so we supplied threonine to the mutant cultures. Effects of threonine on growth were similar to those of aminoacetone (data not shown). To confirm that the accumulation of threonine-derived aminoacetone reduces the growth of $S$. oligofermentans, we first constructed a threonine dehydrogenase gene $(t d h)$ deletion mutant. Under $\Delta t d h$ background conditions, we readily obtained an $a a_{S o}$-mut $\mathrm{T}$ double gene mutant; however, no such mutant was ever made from the wild-type strain as mentioned above. Again this demonstrates that threoninederived aminoacetone is the deleterious compound that needs LAAO to remove. In addition, the triple mutant $\Delta t d h-\Delta a a o_{S o^{-}}$ $\triangle m u t T$ exhibited a growth reduction of only $10 \%$ relative to the wild-type strain (Fig. S6). This suggests that the partial growth impairment of $\Delta a a_{0} o_{S o}-m u t T$ strain is caused by the accumulation of aminoacetone.

\section{Discussion}

In this study, we used genetic and biochemical approaches to demonstrate that the gene operon $a a o_{S_{0}}-m u t T$ constitutes a dual safeguard mechanism, protecting cells from ROS stress in $S$. oligofermentans. Using $a^{a} o_{S o}$-encoded aminoacetone oxidase, cells can reduce the generation of aminoacetone-derived superoxide anion, and the coexpressed MutT eliminates the mutagens derived from ROS. Concerted action of the two proteins can be modulated by the equivalent expression of two genes under the control of a single promoter. This anti-ROS defense mechanism may also be employed by other streptococci. A similar gene operon has also been found in Streptococcus pneumoniae, a streptococcus inhabiting in human upper respiratory tract [25]. However, this operon has not yet been found in other lactic acid bacteria whose genomes are available.

Table 3. Effect of aminoacetone on the growth of S. oligofermentans wild-type and two mutants.

\begin{tabular}{|c|c|c|c|c|c|c|}
\hline \multicolumn{7}{|c|}{ Growth mass $\left(O D_{600}\right)^{*}$} \\
\hline \multirow[b]{2}{*}{ Growth time } & \multicolumn{2}{|c|}{ wild-type } & \multicolumn{2}{|c|}{$\Delta a a o_{s o}$} & \multicolumn{2}{|c|}{$\Delta a a o_{s o}-m u t T$} \\
\hline & - & + & - & + & - & + \\
\hline $6 \mathrm{~h}$ & $0.46 \pm 0.01$ & $0.51 \pm 0.02$ & $0.44 \pm 0.02$ & $0.49 \pm 0.03$ & $0.33 \pm 0.01$ & $0.29 \pm 0.02$ \\
\hline $8 \mathrm{~h}$ & $0.98 \pm 0.03$ & $1.05 \pm 0.03$ & $0.96 \pm 0.03$ & $0.96 \pm 0.04$ & $0.78 \pm 0.01$ & $0.71 \pm 0.01$ \\
\hline
\end{tabular}

*Symbol: +, $5 \mathrm{mM}$ aminoacetone added; -, no aminoacetone added; Data are shown as means \pm SD from three independent experiments.

doi:10.1371/journal.pone.0038133.t003 
Previous research has shown that aminoacetone, a prooxidant, rapidly reacts with oxygen through a superoxide-propagated mechanism even in the presence of strong chelators [26], while semicarbazide-sensitive amine oxidase (SSAO) functions as a cleaner of aminoacetone in human cells [15]. Considering that (1) both LAAO and SSAO belong to the family of amine oxidases; (2) amino acids can also be classified as amines; and (3) aminoacetone is an analog of amino acids; we tested the aminoacetone oxidase activity of LAAO. Pronounced aminoacetone oxidase activity was detected for LAAO, although it was first identified as an amino acid oxidase [11]. Cellular aminoacetone can be derived either from threonine or a condensation of glycine and acetyl-CoA [24,27]. Threonine dehydrogenase, a key enzyme in L-threonine metabolism, catalyzes the conversion of L-threonine to 2-amino-3-ketobutyrate, and the latter is spontaneously decarboxylated to aminoacetone [28]. No gene homology of 2-amino-3-ketobutyrate coenzyme A ligase, which catalyzes glycine and acetyl-CoA in the formation of 2-amino-3-ketobutyrate, was found in the genome of $S$. oligofermentans. However, the $t d h$ gene was present and an elevated transcription level was detected in both $\Delta a a o_{S o}-m u t T$ and $\Delta a a o_{S o}$ mutants (data not shown). This increased aminoacetone production and decreased scavenging. In addition, the $a a_{S_{0}}-m u t T$ double gene mutant was only obtained using a background of $t d h$ mutation, indicating that aminoacetone is derived from threonine can accumulate to lethal levels when both $a a o_{S o}$ and $m u t T$ are absent. Because aminoacetone can react with transition metals, it does not accumulate to a great extent in the cells. This is also true of human cells [23]. The reason why $a a_{S o}$ inactivation induces expression of $t d h$ remains unknown.

It is known that ROS can oxidize nucleotides [19,29]. Among nucleotides, dGTP is most susceptible to oxidation, especially to hydroxyl radicals, and the oxidized product 8-oxo-dGTP causes mutagenesis and carcinogenesis $[18,19]$. Organisms have evolved many different approaches to dealing with mutagenesis. These include the MutT protein in E. coli and hMTH-1 protein in humans, both of which are known to hydrolyze 8-oxo-dGTP to the non-harmful 8-oxo-dGMP and PPi [19,30]. MutT belongs to the Nudix hydrolase superfamily, which requires $\mathrm{Mg}^{2+}$. These enzymes hydrolyze the nucleoside diphosphate modified with other moieties. The most suitable substrate for E. coli MutT is 8oxo-dGTP [31]. In the present study, similar types of activity were determined for $S$. oligofermentans MutT. The high levels of expression of mut $T$ in living $S$. oligofermentans cells and the enzymatic activity of MutT suggest that $S$. oligofermentans MutT also plays a role in the elimination of deleterious oxidized nucleotides.

Although the $\triangle a a 0_{S o}$ mutant generates hydroxyl radicals from superoxide anion and $\mathrm{H}_{2} \mathrm{O}_{2}$, the intact mut $\mathrm{T}$ may be sufficient to the scavenging nucleotide oxides generated by hydroxyl radicals. This may be why no impairment of growth was observed. Under normal growth conditions, the production of ROS is limited to a very low level, thus, $\Delta m u t T$ did not show any detectable reduction in growth. However, inactivation of both $a a o_{S_{o}}$ and $m u t T$ is lethal for S. oligofermentans. Therefore, synergy of LAAO and MutT is essential for bacterial defense against ROS damage. Here, we propose a working model of LAAO and MutT in prevention of ROS-generated cell oxidative damage (Fig. 4).

The $a a o_{S o}-m u t T$ operon and possible coexpression are found in the genome of $S$. pneumoniae (GenBank accession No. NG_008533). This clinical streptococcus resembles $S$. oligofermentans with respect to $\mathrm{H}_{2} \mathrm{O}_{2}$ production and tolerance $[10,25]$. Like $S$. pneumoniae, which lives in an oxygen-rich niche in the upper respiratory tract, S. oligofermentans inhabits the human oral cavity, where the oxygen content fluctuates $[25,32,33]$. In this way, the $a a_{0_{S o}}-m u t T$ operon may have been the evolutionarily selected by bacteria living in environments rich in oxygen.

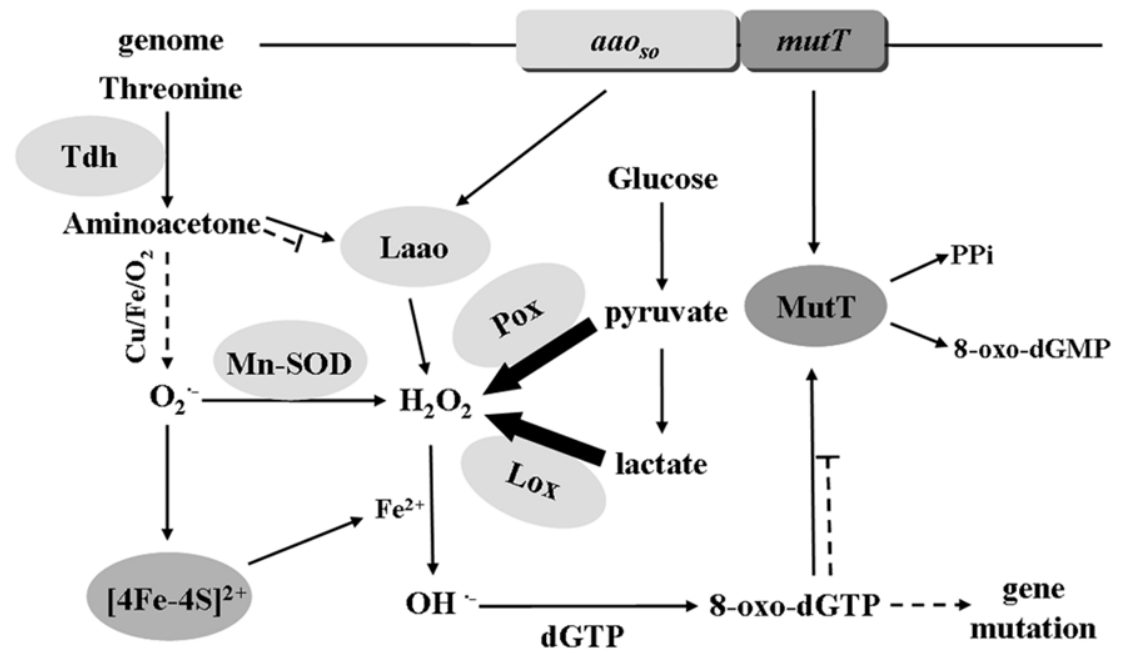

Figure 4. Depicted concerted working model of LAAO and MutT in preventing ROS-generated oxidative damage to cells. $a a O_{S o}$ and mutT constitute an operon in S. oligofermentans and are co-transcribed, creating the equivalent protein products of the two. The absence of LAAO, an aminoacetone oxidase, was found to cause the accumulation of aminoacetone, which can be derived from threonine by threonine dehydrogenase (Tdh). It generates superoxide anions in the presence of oxygen and transition metals. Fe ${ }^{2+}$, released from proteins containing $[4 \mathrm{Fe}-4 \mathrm{~S}]^{2+}$ cluster by superoxide anion attack, was found to trigger the Fenton reaction to form hydroxyl radicals from $\mathrm{H}_{2} \mathrm{O}_{2}$, which is produced in abundance from lactate and pyruvate by Lox and Pox, respectively. These hydroxyl radicals oxidize nucleic acids, like dGTP to 8-oxo-dGTP, a mutagen that can cause severe gene mutations. The coexpressed MutT hydrolyzes 8-oxo-dGTP to the harmless 8-oxo-dGMP, and so prevents cell damage. LAAO, L-amino acid oxidase; MutT, pyrophosphohydrolase; Tdh, threonine dehydrogenase; Lox, lactate oxidase; Pox, pyruvate oxidase; Mn-SOD, manganese-dependent superoxide dismutase. Thick arrows refer to the main flux of $\mathrm{H}_{2} \mathrm{O}_{2}$; broken arrows indicate the conditional pathways. doi:10.1371/journal.pone.0038133.g004 


\section{Materials and Methods}

\section{Bacterial strains and Culture Conditions}

S. oligofermentans strain AS $1.3089^{\mathrm{T}}$ and the $a a_{0} o_{S_{0}}$-insertional mutant were cultured in our laboratory [9,11]. Strains were routinely cultured in TYG broth at $37^{\circ} \mathrm{C}$ in static culture [34]. Agar plates were cultured in a candle jar. Brain heart infusion (BHI) medium (Becton Dickinson, U.S.) supplemented with spectinomycin $\left(800 \mu \mathrm{g} \mathrm{ml}^{-1}\right)$ or kanamycin $\left(500 \mu \mathrm{g} \mathrm{ml}^{-1}\right)$ was used for selection of antibiotic-resistant colonies. E. coli DH5 $\alpha$ (Transgen Biotech, Beijing, China) and BL21 (Novagen, Germany) were grown in Luria-Bertani (LB) broth or on LB agar plate aerobically at $37^{\circ} \mathrm{C}$. They were used for plasmid amplification and protein overexpression. Transformants were selected in $\mathrm{LB}$ medium with ampicillin $\left(100 \mu \mathrm{g} \mathrm{ml}^{-1}\right)$, kanamycin $(50 \mu \mathrm{g} \mathrm{ml}$ $\left.{ }^{1}\right)$, or spectinomycin $\left(250 \mu \mathrm{g} \mathrm{ml}^{-1}\right)$.

\section{Construction of $a a_{S o}$, mutT, and sodA Gene Deletion Mutants}

The genome DNA of S. oligofermentans was extracted and purified as described previously [33,35]. PCR primers listed in Table 4 were designed according to the genome draft of S. oligofermentans and synthesized by Sangon Company (Shanghai, China). The deletion mutants of the $a a o_{S o}$, mut $\mathcal{T}$, and sodA genes were constructed using a PGR ligation method [36]. Two $600 \mathrm{bp}$ sequences upstream and downstream of each of the three genes were PCR amplified using the primers listed in Table 4. PCR products were digested with BamHI (New England Biolabs, U.S.) and a non-polar kanamycin resistance gene cassette was cut from plasmid pALH124 with BamHI as well [36]. The three fragments at equivalent molar ratios were ligated using $\mathrm{T}_{4}$ DNA ligase (Fermentas, Canada). The fused fragment was transformed into wild-type $S$. oligofermentans with the assistance of a chemical synthetic $S$. oligofermentans- specific competence-stimulating peptide (CSP) [33]. Briefly, overnight culture of S. oligofermentans was diluted 1:30 into fresh $\mathrm{BHI}$ broth (with $0.4 \% \mathrm{BSA}$ ) and cultured at $37^{\circ} \mathrm{C}$ for $30 \mathrm{~min}$. The fused fragment was added at a final concentration of $500 \mu \mathrm{g} \mathrm{ml} \mathrm{m}^{-1}$ together with the CSP (final concentration $160 \mathrm{ng} \mathrm{ml}^{-1}$ ). After $5 \mathrm{~h}$ incubation, the mixture was plated on BHI agar containing the selective antibiotics and incubated at $37^{\circ} \mathrm{C}$ in a candle jar. A sodA deletion of $\Delta a a_{0} s_{o}-m u t T$ mutant was constructed using the same method. Positive transformants of the $\Delta a a_{0} o_{s o}-m u t T-\Delta$ sodA mutant were selected on BHI agar plates containing $500 \mu \mathrm{g} \mathrm{ml}^{-1}$ kanamycin and $800 \mu \mathrm{g}$ $\mathrm{ml}^{-1}$ spectinomycin. Transformants of $\Delta a a_{0}, \Delta m u t T$, and $\Delta s o d A$ were selected with $500 \mu \mathrm{g} \mathrm{ml}^{-1}$ each of kanamycin. They were all confirmed by PCR.

\section{Construction of $a a_{S_{0}}-$ mutT and $t d h-a a_{S_{0}}-m u t T$ Deletion Mutants}

To delete the $a a 0_{S o}-m u t T$ genes on the chromosome, we first constructed a shuttle plasmid, pDL278- $a a_{S_{S o}}-m u t T$. The entire $a a_{S o}-m u t T$ operon fragment (including the promoter region) was amplified with paired primers pDL-lm-F and pDL-lm-R (Table 4). The fragment was then digested with BamHI and SalI and inserted into plasmid pDL278, which was digested with the same enzymes. The recombinant plasmid was transformed into the wild-type strain of S. oligofermentans, and the positive strain was selected on BHI agar containing spectinomycin $\left(800 \mu \mathrm{g} \mathrm{ml}^{-1}\right)$. Upon PCR confirmation of the introduced plasmid-carried $a a_{S_{S o}}-m u t T$ operon, a deletion of $a a_{S_{S o}}-m u t T$ operon on the chromosome was conducted using the same method as the single gene mutation above except that the positive transformants were selected on BHI agar plates containing $500 \mu \mathrm{g} \mathrm{ml}^{-1}$ kanamycin and $800 \mu \mathrm{g} \mathrm{ml}^{-1}$ spectinomycin.

To produce the triple-gene $t d h-a a_{S o}-m u t T$ deletion mutant, the $t d h$ gene was knocked out to stop the generation of aminoacetone. Briefly, two 600 bp sequences upstream and downstream of the tdh gene were PCR amplified using the corresponding primers listed in Table 4. PCR products were double digested with HindIII and SmaI (New England Biolabs, U.S.) and a spectinomycin resistance gene cassette was cut from plasmid pFW5-luc with HindIII and SmaI [33,37]. The three fragments at equivalent molar ratio were ligated using $\mathrm{T}_{4}$ DNA ligase (Fermentas, Canada), and the fused fragment was transformed into wild-type $S$. oligofermentans. $\Delta t d h$ mutant was selected on BHI agar plate containing $800 \mu \mathrm{g}$ $\mathrm{ml}^{-1}$ spectinomycin. Under $\Delta t d h$ background, the $a a_{S_{0}}-m u t T$ operon was knocked out. The triple gene deletion $\Delta t d h-\Delta a a_{0} \mathrm{So}^{-}$ $\Delta m u t T$ was selected on BHI agar plate containing $500 \mu \mathrm{g} \mathrm{ml}^{-1}$ kanamycin and $800 \mu \mathrm{g} \mathrm{ml}^{-1}$ spectinomycin. The positive clones were confirmed by PCR.

\section{Construction of $a^{a o_{S o}}$-mutT Gene Complementary Strain}

The $a a o_{S o}-m u t T$ coding region and its inherent promoter were amplified using the paired primers pDL-lm-F and pDL-lm-R (Table 4). The PGR product was double digested with Bam HI and SalI and inserted into plasmid pDL278, which was digested with the same enzymes. Recombinant plasmid pDL278- $a a 0_{S o}-m u t T$ was transformed into the $\Delta a a_{0} o_{s o}-m u t T$ mutant; transformants were selected on BHI agar containing spectinomycin $\left(5 \mathrm{mg} \mathrm{ml}^{-1}\right)$ under aerobic conditions. Positive transformants were confirmed by PCR and sequencing.

Quantification of Gene Expression with Quantitative PCR

Total RNA was extracted using TRIzol reagent (Invitrogen, U.S.) according to the supplier's recommendations. Cells were harvested at the middle logarithmic phase, washed with RNasefree water, and frozen in liquid nitrogen, and then ground three times with a glass rod. Then TRIzol reagent was added. After incubation at room temperature for $5 \mathrm{~min}$, chloroform was mixed with the lysate and the mixture centrifuged at $12,000 \times \mathrm{g}$. RNA in the aqueous phase was then precipitated using isopropyl alcohol, washed with $75 \%$ ethanol, and air dried for $15 \mathrm{~min}$. The pellet was then dissolved in $30 \mu \mathrm{l}$ RNase-free water, and trace amounts of DNA were removed by DNase. The concentration and quality of RNA samples were determined using NanoDrop spectrophotometer and gel electrophoresis. cDNA was synthesized from $2 \mu \mathrm{g}$ RNA according to manufacturer's instructions (Promega, U.S.) and used for quantitative PCR amplification with the corresponding primers listed in Table 4. Amplifications were performed with ABI PRISM 7000 Sequence Detection System (Applied Biosystems, U.S.). The thermocycling parameters included one cycle of $95^{\circ} \mathrm{C}$ for $10 \mathrm{~s}$, followed by 40 cycles of $95^{\circ} \mathrm{C}$ for $5 \mathrm{~s}, 51^{\circ} \mathrm{C}$ for $30 \mathrm{~s}$, and $72^{\circ} \mathrm{C}$ for $30 \mathrm{~s}$. Transcripts were quantified with the comparative threshold cycle $(\mathrm{Ct})$ values. To estimate the copy numbers for a given mRNA, a standard curve of the tested gene was generated by cloning the corresponding PCR fragment at a length of about 260 bp into pMD-18T vector (TaKaRa, Japan). The plasmid was then serially diluted and used to generate the standard curve of quantitative PCR. The $16 \mathrm{~S}$ rRNA gene was used as the biomass reference. The copy number of each gene was normalized to $16 \mathrm{~S}$ rRNA copies. The copies of the transcript of each gene are shown as per 10,000 16S rRNA copies.

\section{Overexpression and Purification of MutT Protein}

The $m u t T$ gene of $S$. oligofermentans was amplified using a pair of primers (Table 4). Pyrobest DNA polymerase (TaKaRa, Japan) 
Table 4. Primers used in this study.

\begin{tabular}{|c|c|c|}
\hline Primer & Sequence* & Target genes \\
\hline spacer-Im-F & TCTCCACTTTGCCGGTGA & spacer between $a a_{\text {So }}$ and mutT \\
\hline spacer-Im-R & CATGCACATGTTGACAAATTC & \\
\hline spacer-neg-F & TCATCAAATAATTGGGCAAGC & negative control for space check \\
\hline spacer-neg-R & ACAAACTATTGACAGCGCTATCAC & \\
\hline qPCR-16S-F & GGCGTGCCTAATACA & qPCR for 165 rRNA \\
\hline qPCR-16S-R & AGACTTCCGTCCATT & \\
\hline qPCR-Sod-F & ATGGCAATTATTTTACCAGATCTAC & qPCR for sodA \\
\hline qPCR-Sod-R & TTCTTCGAATGAGCCAAATG & \\
\hline qPCR-mutT-F & GAATTTGTCAACATGTGCATGA & qPCR for mutT \\
\hline qPCR-mutT-R & CGGTTGCAAGTTTCAGATGA & \\
\hline qPCR-mutX-F & ATTCGTAAAAATCAACCTGCG & qPCR for mutX \\
\hline qPCR-mutX-R & TTTCCTGAGTTTACTCCCGA & \\
\hline mutT-kana-up-F & GAAGAAAAAGGAGAAAGAAAG & upstream fragment of mut $T$ for deletion \\
\hline mutT-kana-up-R & AAGGATCCAAAATGAGAGTGGGACAG & \\
\hline mutT-kana-down-F & AAGGATCCCTTGTTCTCCCTITAAAT & downstream fragment of mut $T$ for deletion \\
\hline mutT-kana-down-R & TGCTGAAAGTCCTCTTTTGAC & \\
\hline$a a o_{s o}-$ kana-up-F & ATGAACCATTTCGACACAATC & upstream fragment of $a a_{s o}$ for deletion \\
\hline$a a o_{s o}$-kana-up-R & TTGGATCCTGTCATGGTTGTCAAAC & \\
\hline$a a_{s o}-$ kana-down-F & TTGGATCCAGGCCCTCATCAAGCAAA & downstream fragment of $a a_{s o}$ for deletion \\
\hline$a a o_{s o}-$ kana-down-R & TTAATCATAATGCAAACTTC & \\
\hline pDL-Im-F & ATGGATCCTAAAAAATGAGCCTAAAATC & construction of shuttle plasmid \\
\hline pDL-Im-R & TATGTCGACTTITTACTTCAAGTCATAAA & \\
\hline Im-kana-up-F & GAAGAAAAAGGAGAAAGAAAG & upstream fragment of $a a_{S o}-m u t T$ for deletion \\
\hline Im-kana-up-R & AAGGATCCAAAATGAGAGTGGGACAG & \\
\hline Im-kana-down-F & AAGGATCCGTATCTTITCCTITCGTG & downstream fragment of $a a_{s_{0}}-m u t T$ for deletion \\
\hline Im-kana-down-R & CAGAGGTCAAGCAGCCCTTG & \\
\hline tdh-add9-up-F & GGATGCGACTGGCAATGCCAAAGAT & upstream fragment of $t d h$ for deletion \\
\hline tdh-add9-up-R & TTAAGCTTTCCTTTTCCTITCCAAATGAG & \\
\hline tdh-add9-down-F & CTTGACTTCTGCATCTGGCTCAAC & downstream fragment of $t d h$ for deletion \\
\hline tdh-add9-down-R & TTTCCCGGGTCCTAAAAATATTTAAGC & \\
\hline sod-kana-up-F & GGTTTGCCCAGTCGTCAGGTC & upstream fragment of sodA for deletion \\
\hline sod-kana-up-R & TTGGATCCGAACTCTACGCAGC & \\
\hline sod-kana-down-F & TTGGATCCGATCTGGTAAAATAA & downstream fragment of sodA for deletion \\
\hline sod-kana-down-R & CAATATTITGATTTGACCCAGCTG & \\
\hline pET-mutT-F & TTCCATGGGGATGAACAGACGCGAAACC & overexpression of MutT \\
\hline pET-mutT-R & TTCTCGAGCTTCAAGTCATAAAGC & \\
\hline
\end{tabular}

*Underlined sequences indicate the restriction enzyme sites. All primers are original in this study.

doi:10.1371/journal.pone.0038133.t004

was used for PCR amplification of mutT. A 453 bp fragment was subsequently digested with $\mathcal{N} c o \mathrm{I}-\mathrm{X} h \mathrm{I}$ (New England Biolabs, U.S.) and cloned into the $\mathcal{N} c o \mathrm{I}-\mathrm{Xho} \mathrm{I}$ restriction sites of the expression vector pET-28a (Novagen, Germany). The His6-tag fusion sites and nucleotide sequences of the double-stranded template DNA were confirmed by sequencing.

The pET-28a plasmid carrying the mut $T$ gene was transformed into E. coli BL21 (DE3) pLysS (Novagen, Germany) and cultured in $\mathrm{LB}$ medium supplemented with $50 \mu \mathrm{g} \mathrm{ml}^{-1}$ kanamycin. Cells were grown at $37^{\circ} \mathrm{C}$ to $\mathrm{OD}_{600} 0.4-0.6$. Overexpression of Mut $\mathrm{T}$ protein was induced by addition of $1 \mathrm{mM}$ isopropyl- $\beta$-D- thiogalactopyranoside. The culture was incubated for an additional $3 \mathrm{~h}$ before harvesting. Cells were collected by centrifugation at $10,000 \times \mathrm{g}$ for
$10 \mathrm{~min}$ at $4^{\circ} \mathrm{C}$, resuspended in the binding buffer $(20 \mathrm{mM}$ sodium phosphate, $0.5 \mathrm{M} \mathrm{NaCl}, 40 \mathrm{mM}$ imidazole, $\mathrm{pH} 7.4$ ), and was sonicated for $20 \mathrm{~min}$. After the cell lysate was spun down at 10,000 $\times \mathrm{g}$ for $10 \mathrm{~min}$ at $4^{\circ} \mathrm{C}$, the supernatant was filtered through a $0.22 \mu \mathrm{m}$ polyvinylidene difluoride membrane (Millipore, U.S.) and then applied to a Histrap column (GE Healthcare, USA) previously equilibrated with the binding buffer. Proteins were eluted by elution buffer (20 mM sodium phosphate, $0.5 \mathrm{M} \mathrm{NaCl}, 500 \mathrm{mM}$ imidazole, $\mathrm{pH} 7.4$ ), and fractions of the elution were subjected to $12 \%$ SDS-PAGE. The desired protein fractions were desalted by loading onto HiPrep $^{\text {TM }}$ 26/10 Desalting (GE Healthcare, U.S.) column and eluted with $20 \mathrm{mM}$ Tris-HCl buffer (pH 9.0) with $10 \%$ glycerol. The purified MutT protein was stored at $-80^{\circ} \mathrm{C}$. 


\section{Assay of Aminoacetone Oxidase Activity for LAAO}

The aminoacetone activity of LAAO was determined by measuring $\mathrm{H}_{2} \mathrm{O}_{2}$ production via horseradish peroxidase (HRP)coupled assay [11]. The assay mixture $(1 \mathrm{ml})$ contained $200 \mathrm{nM}$ LAAO and $25 \mathrm{mM}$ aminoacetone (Toronto Research Chemicals, Canada) in $20 \mathrm{mM}$ Tris-HCl buffer (pH 8.0). Lysine at concentrations of $25 \mathrm{mM}$ and $100 \mathrm{mM}$ was used as the control. The reaction mixture was incubated at $37^{\circ} \mathrm{C}$ for $30 \mathrm{~min} . \mathrm{H}_{2} \mathrm{O}_{2}$ was quantified using a modified version of a previously described method $[11,38]$. Briefly, $650 \mu \mathrm{l}$ of reaction supernatant was added to $600 \mu \mathrm{l}$ of the solution containing $2.5 \mathrm{mM} 4$-amino-antipyrine (4-amino-2, 3-dimethyl-1-phenyl- 3-pyrazolin-5-one; Sigma-Aldrich, U.S.) and $0.17 \mathrm{M}$ phenol. The reaction proceeded for 4 min at room temperature, and HRP (Sigma-Aldrich, U.S.) was added at a final concentration of $50 \mathrm{mU} \mathrm{ml}^{-1}$ in $200 \mathrm{mM}$ potassium phosphate buffer ( $\mathrm{pH}$ 7.2). After $4 \mathrm{~min}$ of incubation at room temperature, $\mathrm{OD}_{510}$ was measured using a Unico 2100 visible spectrophotometer (Shanghai, China). A standard curve was generated with $\mathrm{H}_{2} \mathrm{O}_{2}$.

\section{Mn-SOD Activity Assay}

The Mn-SOD activity of S. oligofermentans was determined using the method of inhibition of pyrogallol auto-oxidation [39,40]. The reaction mixtures included $4.5 \mathrm{ml} 50 \mathrm{mM}$ Tris- $\mathrm{HCl}(\mathrm{pH} \mathrm{8.2)}$, $1 \mathrm{mM}$ EDTA, $0.1 \mathrm{mM}$ pyrogallol, and $10 \mu \mathrm{l}$ freshly prepared lysate supernatant of the strains. The same volume of $10 \mathrm{mM} \mathrm{HCl}$ was used as a blank control. After mixing, $\mathrm{OD}_{325}$ was read every $30 \mathrm{~s}$ for $4 \mathrm{~min}$ at $25^{\circ} \mathrm{C}$ using a Unico 2100 visible spectrophotometer. The $\mathrm{OD}_{325}$ increase rate was calculated from 0 to $4 \mathrm{~min}$. One unit of enzymatic activity was defined as the amount of enzyme used for $50 \%$ reduction of pyrogallol auto-oxidation rate per min per mg protein.

\section{S. oligofermentans MutT Activity Assay}

The enzymatic activity of $S$. oligofermentans Mut T was determined based on the 8-oxo-dGTP degradation. The assay mixture contained $100 \mu \mathrm{l} 100 \mathrm{mM}$ Tris-HCl (pH 9.0), $5 \mathrm{mM} \mathrm{MgCl}_{2}$, $100 \mu \mathrm{M}$ 8-oxo-dGTP (eENZYME, U.S.), 5\% glycerol and $100 \mathrm{nM}$ MutT protein. Desalting buffer without enzyme was used as a negative control. The reaction mixtures were incubated at $37^{\circ} \mathrm{C}$ for $20 \mathrm{~min}$, and terminated by adding $50 \mathrm{mM}$ EDTA (EDTA: $\mathrm{Mg}^{2+}=3.3: 1$ ). Twenty microliters of sample was analyzed on HPLC with the following parameters, flow phases: $20 \mathrm{mM}$ sodium phosphate buffer ( $\mathrm{pH}$ 6.0) and acetonitrile (95:5);

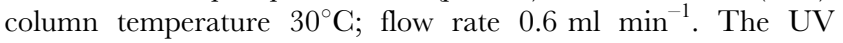
absorbance of nucleotide was detected at $293 \mathrm{~nm}$.

\section{Determination of Cellular Aminoacetone Derivate}

Cells of S. oligofermentans wild-type and two mutants at their mid$\log$ phase were collected and washed twice with $100 \mathrm{mM}$ sodium phosphate buffer $(\mathrm{pH} 7.3)$ by centrifugation at $12,000 \times \mathrm{g}$ for $10 \mathrm{~min}$. Fifty milligrams of pelleted cells of each culture were resuspended in $1 \mathrm{ml} 100 \mathrm{mM}$ phosphate buffer $(\mathrm{pH} 7.3)$, then homogenized in a MINI Bead Beater (Biospec Products; Bartlesville, OK, U.S.) for $20 \mathrm{~s}$, with a 2-min interval on ice. This process was repeated five times. After centrifugation of the cell extract at $12,000 \times \mathrm{g}$ for $10 \mathrm{~min}$ at $4^{\circ} \mathrm{C}$, the supernatant was partially purified using a $3 \mathrm{KD}$ ultrafiltration tube (MWCO, Millipore, U.S.). Synthetic aminoacetone (Toronto Research Chemicals, Canada) and the cell extract samples were treated with FMOC-Cl to obtain the aminoacetone derivate using a modified version of the method described by Kazachkov and $\mathrm{Yu}$ [23]. Briefly, $1 \mathrm{ml}$ sodium borate buffer $(0.4 \mathrm{M}, \mathrm{pH} 10)$ was added to $1 \mathrm{ml}$ of samples or aminoacetone $(10 \mathrm{mM})$ and vortexed for $1 \mathrm{~min}$. Then, $1 \mathrm{ml} \mathrm{FMOC-Cl}$ reagent $(10 \mathrm{mM}$ in acetonitrile) was mixed with the samples by vortex for $1 \mathrm{~min}$. Five milliliters of hexane was added to terminate the reaction by extracting the excess reagent (FMOC-Cl), hydrolysis product FMOC-OH and acetonitrile. The upper hexane layer was discarded and the process was repeated twice, and the remaining solution was neutralized by addition of $100 \mu \mathrm{l} 20 \%(\mathrm{v} / \mathrm{v})$ acetic acid for HPLC assay using a modified version of the method described by Xiao and $\mathrm{Yu}$ [41]. The assay was performed on Shimadzu HPLC system (Japan) with a $\mathrm{C}_{18}$ reverse phase column $(4.6 \times 250 \mathrm{~mm} /$ $5 \mu \mathrm{m}$; Agilent, U.S.), and the compound separation was achieved under the following conditions, flow phases: $5 \mu \mathrm{M}$ potassium phosphate buffer ( $\mathrm{pH} 4.40-4.45)$ and acetonitrile (60:40), column

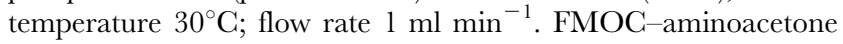
was detected for UV absorbance at $265 \mathrm{~nm}$.

\section{Determination of ROS Levels}

Cellular ROS levels in S. oligofermentans were measured using a spin trapping agent combined with ESR spectroscopy (Bruker ESP 300, Germany) [21,22]. 5,5-Dimethyl-l-pyrroline $\mathcal{N}$-oxide (DMPO, TCI, Japan) was purified by stirring a solution $(0.25 \mathrm{M})$ of the spin trap in deionized water with a little activated charcoal for 1 hour. After filtration, this solution was stored in the dark at $-20^{\circ} \mathrm{C}$. The wild-type strain and two mutants were collected at mid-log phase and washed twice with sodium sulfate solution $(50 \mathrm{mM})$. The standard incubation consisted of the following final concentrations: $30 \mathrm{mg}$ bacterial cells, DMPO solution $(100 \mathrm{mM})$, and each sample were brought up to $0.5 \mathrm{ml}$ with deionized water. The mixtures were incubated at $37^{\circ} \mathrm{C}$ for 5 min before detection. ESR samples in a quartz flat cell were fixed in the cavity of the ESR spectrometer. ESR measurements were taken at $9.75 \mathrm{GHz}$ with a $100 \mathrm{kHz}$ modulation frequency, and the following ESR spin trapping parameters were used: field sweep, 343.2-353.2 mT; microwave frequency, $9.75 \mathrm{GHz}$; sweep time, $168 \mathrm{~s}$; microwave power, $12.8 \mathrm{~mW}$; modulation amplitude $1 \mathrm{mT}$; and time constant, $328 \mathrm{~ms}$.

\section{Supporting Information}

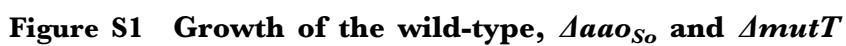
mutants of $\boldsymbol{S}$. oligofermentans in glucose. Strains were precultured overnight in TYG medium, and 1:100 diluted with fresh TYG medium with the same $\mathrm{OD}_{600}$. Samples were taken every $1 \mathrm{~h}$ to detect $\mathrm{OD}_{600}$. Symbols: $\triangle$, wild-type strain; $\bigcirc, \Delta a a_{0_{S o}}$ mutant; $\times, \Delta m u t T$ mutant. The results are shown as the means $\pm \mathrm{SD}$ of three independent experiments.

(TIF)

Figure S2 Enzymatic assays for the overexpressed LAAO of S. oligofermentans. Activities are expressed by $\mathrm{H}_{2} \mathrm{O}_{2}$ production rate as described in materials and methods. Data are the means of three assays, and standard deviations are shown.

(TIF)

Figure S3 Pyrophosphohydrolase activity of $S$. oligofermentans MutT for 8-oxo-dGTP determined through HPLC. (A) 8-oxo-dGTP $(100 \mu \mathrm{M})$ and (B) 8-oxo-dGTP incubated with $100 \mathrm{nM}$ purified MutT at $37^{\circ} \mathrm{C}$ for $20 \mathrm{~min}$. Retention times for 8-oxo-dGTP and 8-oxo-dGMP are shown.

(TIF)

Figure S4 Growth of the wild-type, $\Delta a a o_{S o}-\operatorname{mutT}, \Delta s o d$, and $\Delta_{a a o_{S o}}-m u t T-\Delta s o d$ mutants of $S$. oligofermentans. Strains were cultured overnight and adjusted to the same $\mathrm{OD}_{600}$, and then 1:100 diluted with fresh TYG medium and cultured 
statically unless indicated otherwise. $\mathrm{OD}_{600}$ was measured in $1 \mathrm{~h}$ interval. Symbols: $\triangle$, wild-type strain; $\square, \Delta a a 0_{S o}-m u t T$ mutant; $\diamond$, $\Delta$ sod mutant; $\times, \Delta a a o_{S o}-m u t T-\Delta$ sod mutant; $\bigcirc, \Delta a a o_{S o}-m u t T-\Delta$ sod mutant growing strict anaerobically. Results represent the means \pm SD from three independent experiments.

(TIF)

Figure S5 HPLC chromatogram of FMOG-aminoacetone obtained from the wild-type and both mutants cells of $\boldsymbol{S}$. oligofermentans. The FMOC derivate signal of the chemical aminoacetone appears at $27.5 \mathrm{~min}$ under the analytical conditions. (A) FMOC derivate of chemical aminoacetone; (B) wild-type strain; (C) $\Delta a a o_{S o}$ mutant, and (D) $\Delta a a o_{S o}-m u t T$ mutant. (TIF)

\section{References}

1. Farr SB, Kogoma T (1991) Oxidative stress responses in Escherichia coli and Salmonella typhimurium. Microbiol Rev 55: 561-585.

2. Soonsanga S, Lee JW, Helmann JD (2008) Oxidant-dependent switching between reversible and sacrificial oxidation pathways for Bacillus subtilis OhrR. Mol Microbiol 68: 978-986.

3. Spolarics Z, Wu JX (1997) Role of glutathione and catalase in $\mathrm{H}_{2} \mathrm{O}_{2}$ detoxification in LPS-activated hepatic endothelial and Kupffer cells. Am J Physiol 273: G1304-1311.

4. Yesilkaya H, Kadioglu A, Gingles N, Alexander JE, Mitchell TJ, et al. (2000) Role of manganese-containing superoxide dismutase in oxidative stress and virulence of Streptococcus pneumoniae. Infect Immun 68: 2819-2826.

5. Jenney FE, Jr., Verhagen MF, Gui X, Adams MW (1999) Anaerobic microbes: oxygen detoxification without superoxide dismutase. Science 286: 306-309.

6. Barre O, Mourlane F, Solioz M (2007) Copper induction of lactate oxidase of Lactococcus lactis: a novel metal stress response. J Bacteriol 189: 5947-5954.

7. Magnani D, Barre O, Gerber SD, Solioz M (2008) Characterization of the CopR regulon of Lactococcus lactis IL1403. J Bacteriol 190: 536-545.

8. Rodriguez R, Redman R (2005) Balancing the generation and elimination of reactive oxygen species. Proc Natl Acad Sci U S A 102: 3175-3176.

9. Tong H, Gao X, Dong X (2003) Streptococcus oligofermentans sp. nov., a novel oral isolate from caries-free humans. Int J Syst Evol Microbiol 53: 1101-1104.

10. Tong H, Chen W, Merritt J, Oi F, Shi W, et al. (2007) Streptococcus oligofermentans inhibits Streptococcus mutans through conversion of lactic acid into inhibitory $\mathrm{H}_{2} \mathrm{O}_{2}$ : a possible counteroffensive strategy for interspecies competition. Mol Microbiol 63: 872-880.

11. Tong H, Chen W, Shi W, Qi F, Dong X (2008) SO-AAO, a novel L-amino acid oxidase that enables Streptococcus oligofermentans to outcompete Streptococcus mutans by generating $\mathrm{H}_{2} \mathrm{O}_{2}$ from peptone. J Bacteriol 190: 4716-4721.

12. Liu L, Tong H, Dong X (2012) Function of pyruvate oxidase - lactate oxidase cascade in interspecies competition between Streptococcus oligofermentans and Streptococcus mutans. Appl Environ Microbiol. In press.

13. Zhu B, Tong H, Chen W, Dong X (2009) Role of $d p r$ in hydrogen peroxide tolerance of Streptococcus oligofermentans. Wei Sheng Wu Xue Bao 49: 1341-1346.

14. Bialkowski K, Kasprzak KS (1998) A novel assay of 8-oxo-2'-deoxyguanosine 5'triphosphate pyrophosphohydrolase (8-oxo-dGTPase) activity in cultured cells and its use for evaluation of cadmium(II) inhibition of this activity. Nucleic Acids Res 26: 3194-3201.

15. Sartori A, Garay-Malpartida HM, Forni MF, Schumacher RI, Dutra F, et al. (2008) Aminoacetone, a putative endogenous source of methylglyoxal, causes oxidative stress and death to insulin-producing RINm5f cells. Chem Res Toxicol 21: $1841-1850$.

16. Deng Y, Yu PH (1999) Assessment of the deamination of aminoacetone, an endogenous substrate for semicarbazide-sensitive amine oxidase. Anal Biochem 270: 97-102.

17. Buffoni F, Ignesti $\mathrm{G}(2000)$ The copper-containing amine oxidases: biochemical aspects and functional role. Mol Genet Metab 71: 559-564.

18. Kalam MA, Haraguchi K, Chandani S, Loechler EL, Moriya M, et al. (2006) Genetic effects of oxidative DNA damages: comparative mutagenesis of the imidazole ring-opened formamidopyrimidines (Fapy lesions) and 8-oxo-purines in simian kidney cells. Nucleic Acids Res 34: 2305-2315.

19. Nakabeppu Y, Oka S, Sheng Z, Tsuchimoto D, Sakumi K (2010) Programmed cell death triggered by nucleotide pool damage and its prevention by Mut $T$ homolog-1 (MTH1) with oxidized purine nucleoside triphosphatase. Mutat Res 703: 51-58.

20. Imlay JA (2008) Cellular defenses against superoxide and hydrogen peroxide. Annu Rev Biochem 77: 755-776.

21. Clapp PA, Davies MJ, French MS, Gilbert BC (1994) The bactericidal action of peroxides; an E.P.R. spin-trapping study. Free Radic Res 21: 147-167.

\section{Figure S6}

Growth of $\Delta t d h-\Delta a a o_{S o}-\Delta m u t T$ mutant and the wild-type of $\boldsymbol{S}$. oligofermentans. Strains were cultured overnight and adjusted to the same $\mathrm{OD}_{600}$ in TYG medium, and 1:100 diluted with the same medium. $\mathrm{OD}_{600}$ was detected in $1 \mathrm{~h}$ interval. Symbols: $\triangle$, wild-type strain; $\square, \Delta t d h$ - $\triangle a a{ }_{\text {so }}-\Delta$ mut $T$ mutant. The results are shown as the means $\pm \mathrm{SD}$ of three independent experiments.

(TIF)

\section{Author Contributions}

Conceived and designed the experiments: PZ XD HT. Performed the experiments: PZ LL. Analyzed the data: PZ XD HT. Contributed reagents/materials/analysis tools: PZ LL. Wrote the paper: PZ XD HT.

22. Zhou N, Qiu T, Liu YP, Liu Y (2006) Superoxide anion radical generation in the $\mathrm{NaOH} / \mathrm{H}_{2} \mathrm{O}_{2} / \mathrm{Fe}(\mathrm{III})$ system: a spin trapping ESR study. Magn Reson Chem 44: 38-44.

23. Kazachkov M, Yu PH (2005) A novel HPLC procedure for detection and quantification of aminoacetone, a precursor of methylglyoxal, in biological samples. J Chromatogr B Analyt Technol Biomed Life Sci 824: 116-122.

24. Hiraku Y, Sugimoto J, Yamaguchi T, Kawanishi S (1999) Oxidative DNA damage induced by aminoacetone, an amino acid metabolite. Arch Biochem Biophys 365: 62-70.

25. Pericone CD, Bae D, Shchepetov M, McCool T, Weiser JN (2002) Shortsequence tandem and nontandem DNA repeats and endogenous hydrogen peroxide production contribute to genetic instability of Streptococcus pneumoniae. J Bacteriol 184: 4392-4399.

26. Dutra F, Knudsen FS, Curi D, Bechara EJ (2001) Aerobic oxidation of aminoacetone, a threonine catabolite: iron catalysis and coupled iron release from ferritin. Chem Res Toxicol 14: 1323-1329.

27. Marcus JP, Dekker EE (1993) Identity and some properties of the L-threonine aldolase activity manifested by pure 2-amino-3-ketobutyrate ligase of Escherichia coli. Biochim Biophys Acta 1164: 299-304.

28. Bashir Q, Rashid N, Jamil F, Imanaka T, Akhtar M (2009) Highly thermostable L-threonine dehydrogenase from the hyperthermophilic archaeon Thermococcus kodakaraensis. J Biochem 146: 95-102.

29. Taddei F, Hayakawa H, Bouton M, Cirinesi A, Matic I, et al. (1997) Counteraction by MutT protein of transcriptional errors caused by oxidative damage. Science 278: 128-130.

30. Tajiri T, Maki H, Sekiguchi M (1995) Functional cooperation of MutT, MutM and MutY proteins in preventing mutations caused by spontaneous oxidation of guanine nucleotide in Escherichia coli. Mutat Res 336: 257-267.

31. Mildvan AS, Xia Z, Azurmendi HF, Saraswat V, Legler PM, et al. (2005) Structures and mechanisms of Nudix hydrolases. Arch Biochem Biophys 433: 129-143.

32. Marriott HM, Dockrell DH (2006) Streptococcus pneumoniae: the role of apoptosis in host defense and pathogenesis. Int J Biochem Cell Biol 38: 1848-1854.

33. Tong H, Zhu B, Chen W, Oi F, Shi W, et al. (2006) Establishing a genetic system for ecological studies of Streptococcus oligofermentans. FEMS Microbiol Lett 264: $213-219$

34. Scardovi V (1986) Bergey's Manual of Systematic Bacteriology. Baltimore, MD: Williams \& Wilkins.

35. Dong X, Xin Y, Jian W, Liu X, Ling D (2000) Bifidobacterium thermacidophilum sp. nov., isolated from an anaerobic digester. Int J Syst Evol Microbiol 50 Pt 1: 119-125.

36. Ahn SJ, Wen ZT, Burne RA (2006) Multilevel control of competence development and stress tolerance in Streptococcus mutans UA159. Infect Immun 74: 1631-1642.

37. Podbielski A, Woischnik M, Leonard BA, Schmidt KH (1999) Characterization of nra, a global negative regulator gene in group A streptococci. Mol Microbiol 31: 1051-1064.

38. Seki M, Iida K, Saito M, Nakayama H, Yoshida S (2004) Hydrogen peroxide production in Streptococcus pyogenes: involvement of lactate oxidase and coupling with aerobic utilization of lactate. J Bacteriol 186: 2046-2051.

39. Scorei R, Cimpoiasu VM, Iordachescu D (2005) In vitro evaluation of the antioxidant activity of calcium fructoborate. Biol Trace Elem Res 107: 127-134.

40. Siegel D, Gustafson DL, Dehn DL, Han JY, Boonchoong P, et al. (2004) $\mathrm{NAD}(\mathrm{P}) \mathrm{H}$ :quinone oxidoreductase 1: role as a superoxide scavenger. Mol Pharmacol 65: 1238-1247.

41. Xiao S, Yu PH (2009) A fluorometric high-performance liquid chromatography procedure for simultaneous determination of methylamine and aminoacetone in blood and tissues. Anal Biochem 384: 20-26. 